Research Article

\title{
Pipeline Lifting Mechanics Research of Horizontal Directional Drilling
}

\author{
Zhengwei Guo $\mathbb{D}^{D}$, Shuai Xie, Liancheng Ren, Gong Yinchun $(\mathbb{D}$, and Chaoqun Dong
}

Research Institute of Petroleum and Natural Gas Equipment, Chongqing University of Science \& Technology, Chongqing 401331, China

Correspondence should be addressed to Zhengwei Guo; 997364742@qq.com

Received 11 December 2019; Revised 14 March 2020; Accepted 14 May 2020; Published 8 June 2020

Academic Editor: Pablo Lopez-Crespo

Copyright $(92020$ Zhengwei Guo et al. This is an open access article distributed under the Creative Commons Attribution License, which permits unrestricted use, distribution, and reproduction in any medium, provided the original work is properly cited.

During lifting pipeline of horizontal directional drilling (HDD), the rotation angle of pipeline is determined by such parameters as the location of lifting point, axial force, and length of pipeline. The continuous beam theory is used to analyze the mechanical behaviour of pipeline during lifting construction, and the mechanical model of pipeline during lifting construction process is established. The analysis results show that the lifting height of gondola 1 varies linearly with the length of pipeline. The lifting height of gondola 2 varies linearly with axial force and pipe gravity. The longer the spanning pipeline is, the higher the lifting height is. The lifting height of gondola 2 varies curve trend with the axial force and pipe stiffness. When the length of pipeline is small, the lifting height of gondola 2 is approximately 0 .

\section{Introduction}

HDD is a new construction technology that combines the directional drilling well technology of petroleum with the pipeline construction method. This construction method has advantages of fast construction speed, high construction precision, and lower cost. Therefore, it is widely used in laying pipeline $[1,2]$. The HDD technology includes drilling pilot hole, multistage reaming, and pull-back pipeline, among which pull-back pipeline is the most important procedure of pipeline laying construction.

In a construction field of pull-back pipeline, due to the limitation of ground space and construction condition, the way of sending pipeline and the designing of a dragging parameter should be selected to ensure that the pipeline is pullbacked smoothly into the hole. At present, the field construction adopts the way of gondola combined with a delivery ditch to send pipeline, field construction as shown in Figure 1.

During process of lifting pipeline, if improper construction parameters of lifting pipeline make the angle between pipeline's axis and hole's axis exceed the permitted range, it may cause a large contact force between the pipe and the hole. Therefore, it is necessary to strictly control the lifting parameters to ensure thepipeline safety and smoothly into the hole. In the construction field, engineering experience is often adopted to adjust the height of the gondola and the length of spanning pipeline so as to allow the pipeline smoothly into the hole. However, this method lacks theoretical basis, and it is easy to cause a large contact force between the pipe and hole due to wrong judgment. The lifting pipeline construction is shown in Figure 2. Under certain entry angle of the hole, it is of great significance to send the pipeline into the hole if the lifting construction parameters are calculated by a mathematical method.

The pull-back pipeline construction includes two stages of lifting and pull-back pipeline. At present, there is seldom research on lifting pipeline construction, and most of the research is limited to the calculation of pull-back force inside the hole. However, lifting construction outside the hole will affect pipeline's pull-back force, so it is necessary to analyze the mechanical behaviour of lifting pipeline outside the hole. Ariaratnam et al. [3] analyzed the effect of mud on stability of a hole wall. Cheng and Polak [4] established the theoretical model of pull-back pipeline. Puckett [5] used a new theoretical method to calculate the pull-back load in process of pull-back pipeline. Wu et al. [6] 


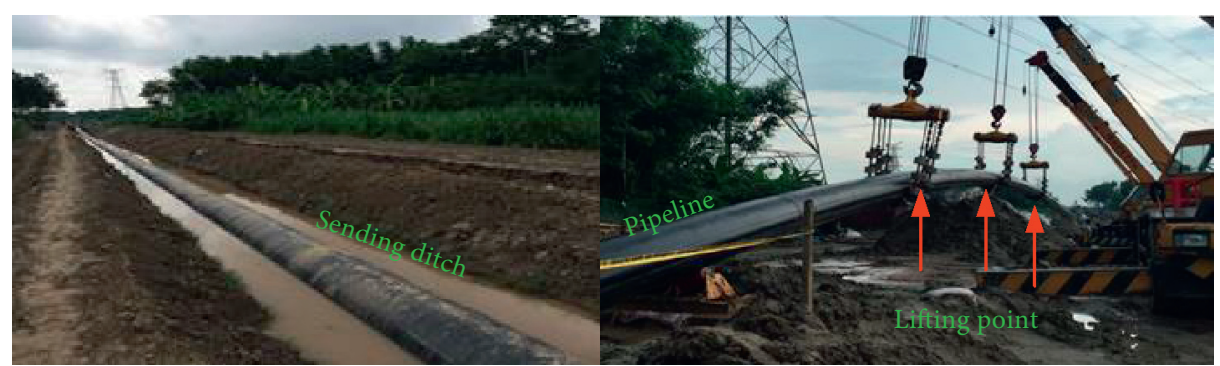

FIGURe 1: Pipeline lifting construction field.

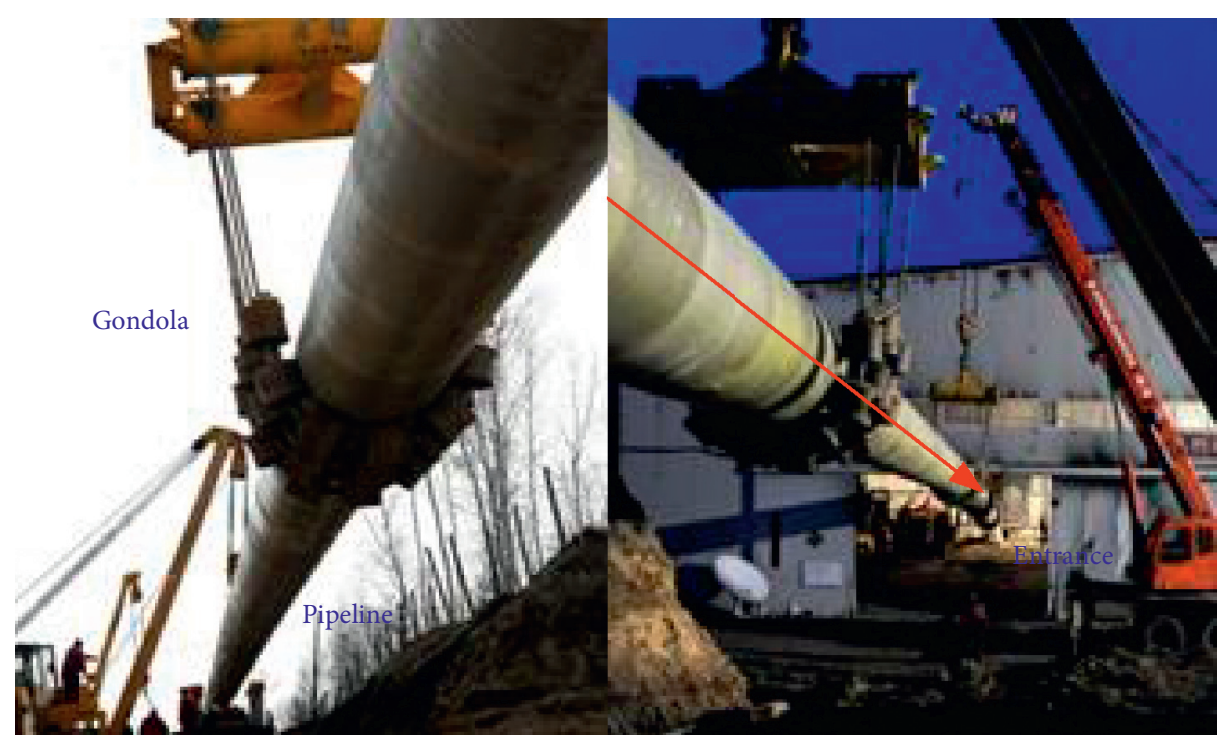

FIgURE 2: Lifting pipeline send into the hole.

tested the pull-back force during laying a submarine cable by an experimental test, which is comparative with theoretical results [6]. Xu et al. [7] numerically simulated the lifting pipeline construction adopted by a numerical calculation method, and the numerical calculation results are compared with the experimental results [7]. Ningping et al [8] described the characteristics and research direction of drilling equipment and analyzed the key equipment of HDD technology [8]. Xia et al. [9] proposed a calculation formula of pull-back force inside the hole, and the theoretical calculation results are compared with the experiment test results [9]. Podbevsek et al. [10] proved that the pipeline has a large reaction force in the curved segment through a theoretical method [10].

This paper analyses the relationship between various parameters during lifting pipeline process by continuous beam theory. The allowable lifting height range and the relationship between various parameters are obtained under the certain entry angle of the hole. The full-scale model of pipeline lifting construction is established by a numerical analysis method to verify the theoretical calculation results. This research provides theoretical basis for the lifting pipeline construction, and it has certain guiding significance for lifting pipeline.

\section{Mechanical Model of Lifting Pipeline}

During the lifting pipeline construction, the number of gondolas is related to the construction space and pipe parameters. Assume that the number of gondolas for lifting pipeline is $n$, and the length of the first gondola to the hole inlet is $L_{1}$, and the length of each spanning pipeline is $L_{i}$ $(i=1,2, \ldots, n)$. The length of the pipeline in sending ditch is $L_{n+2}$, and the length of the last spanning pipeline is $L_{n+1}$. The schematic diagram of the lifting pipeline construction is shown in Figure 3.

If each spanning pipeline is taken as the research object and adjacent spanning pipeline satisfied the continuity condition, assuming the lifting load of each gondola is $F_{i}$, the shear force equations at the gondola position are as follows:

$$
\left\{\begin{array}{c}
E I y_{11}^{\prime \prime \prime}+F_{1}=E I y_{2 n}^{\prime \prime \prime}, \\
\vdots \\
E I y_{i 1}^{\prime \prime \prime}+F_{i}=E I y_{i+1 n}^{\prime \prime \prime}, \\
\vdots \\
E I y_{n 1}^{\prime \prime \prime}+F_{i}=E I y_{n+1 n}^{\prime \prime \prime} \\
\quad i=1,2, \ldots, i, \ldots, n,
\end{array}\right.
$$




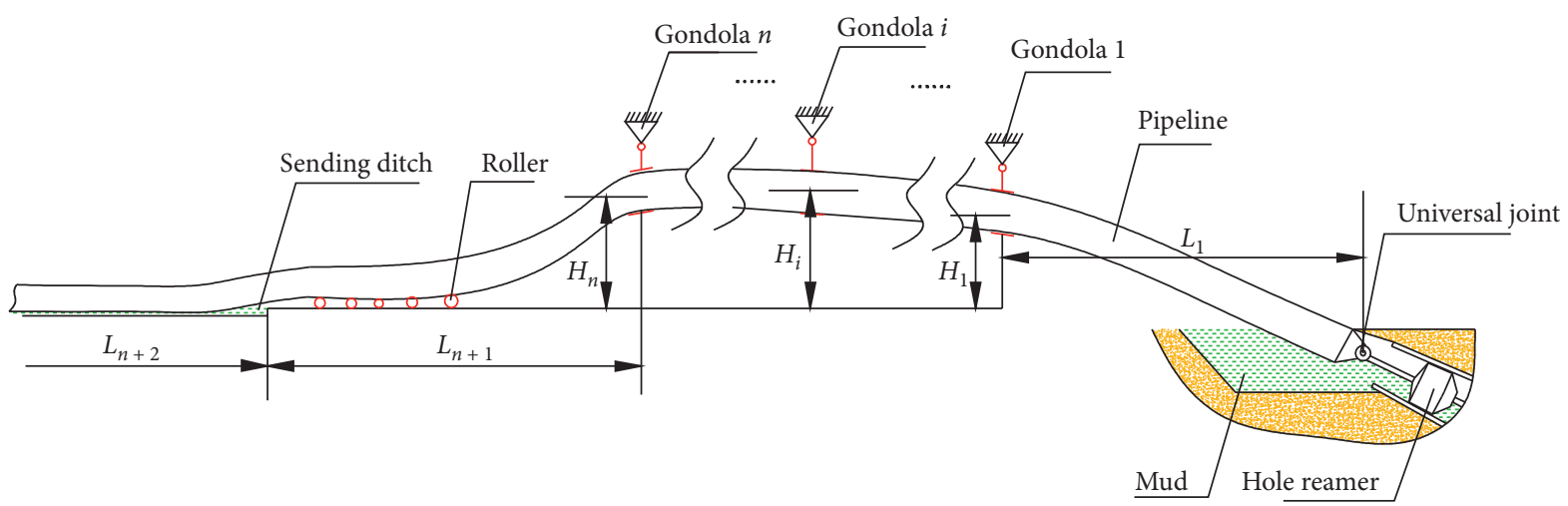

FIgURE 3: Schematic diagram of the lifting pipeline.

where $E I y_{i 1}^{\prime \prime \prime}$ is the shear force of the left end for the $i$ segment spanning pipeline, $\mathrm{N} ; E I y_{i+1 n}^{\prime \prime \prime}$ is the shear force of the right end for the $i+1$ segment spanning pipeline, $\mathrm{N}$; and $E I$ is the stiffness of the pipeline.

Bending moment equations of pipeline are as follows:

$$
\left\{\begin{aligned}
E I y_{11}^{\prime \prime}= & E I y_{2 n}^{\prime \prime}, \\
& \vdots \\
E I y_{i 1}^{\prime \prime}= & E I y_{i+1 n}^{\prime \prime}, \\
\vdots & \\
E I y_{n 1}^{\prime \prime}= & E I y_{n+1 n}^{\prime \prime}, \\
& \quad i=1,2, \ldots, i, \ldots, n .
\end{aligned}\right.
$$

Deflection and rotation angle equations are as follows:

$$
\left\{y_{11}^{\prime}=y_{2 n}^{\prime}, \vdots y_{i 1}^{\prime}=y_{i+1 n}^{\prime}, \vdots y_{n 1}^{\prime}=y_{n+1 n}^{\prime}, \quad\left\{\begin{array}{c}
y_{11}=y_{2 n}, \\
\vdots \\
y_{i 1}=y_{i+1 n} \\
\vdots \\
y_{n 1}=y_{n+1 n} \\
i=1,2, \ldots, i, \ldots, n,
\end{array}\right.\right.
$$

where $E I y_{i 1}^{\prime \prime}$ is the bending moment at the left end for the $i$ segment spanning pipeline, $\mathrm{N} \cdot \mathrm{m} ; E I y_{i+1 n}^{\prime \prime}$ is the bending moment of the right end for the $i+1$ segment spanning pipeline, N.m; $y_{i 1}^{\prime}$ is the rotation angle of the left end for the $i$ segment spanning pipeline, rad; $y_{i+11}^{\prime}$ is the rotation angle of the right end for the $i+1$ segment spanning pipeline, rad; $y_{i 1}$ is the deflection of the left end for the $i$ segment spanning pipeline, $\mathrm{m}$; and $y_{i+11}$ is the deflection of the right end for the $i+1$ segment spanning pipeline, $\mathrm{m}$.

According to the continuity and boundary condition of pipeline in sending ditch, the relationship among the lifting construction parameters can be obtained. In the construction field, two gondolas are widely used for lifting construction. The following analysis is conducted on the pipeline lifting construction taking two gondolas as an example, that is, $n=2$.
2.1. Pipeline Mechanical Model in Sending Ditch. A plane rectangular coordinate system oxy is established as the coordinate system of the entire lifting construction model, and the transition position of the sending ditch and spanning pipeline is the coordinate origin. In infinite distance of pipeline, it satisfies the boundary conditions $y_{\mid x=-L_{4}}^{\prime}=y_{\mid x=-L_{4}}^{\prime \prime}=y_{\mid x=-L_{4}}^{\prime \prime \prime}=0$. It is the free end of the pipeline, and the deflection of the pipeline in sending ditch is equal to the depth of sending ditch $d$.

Take the pipeline in the sending ditch as the research object, and the mechanical model is shown in Figure 4.

Friction force between the unit length of pipeline and soil is $\tau, \tau=\mu q$, and $\mu$ is the friction coefficient between the pipe and soil. The friction coefficient with different materials and soil is shown in Table 1 , and the friction force is uniform distribution along axial direction of the pipeline. Suppose that the length of pipeline in the sending ditch is $L_{4}$, and the depth of the sending ditch is $d$.

The soil's reaction coefficient in the sending ditch is $k_{0}$, and the foundation reaction coefficient is shown in Table 2.

According to the Winkler elastic foundation model, the reaction force of the ground to the pipeline is as follows:

$$
P=-k_{0} D \eta
$$

where $P$ is the reaction force of ground to pipeline; $\eta$ is the pipeline deflection, $\mathrm{m} ; D$ is the pipe diameter, $\mathrm{m}$; and $k_{0}$ is the ground reaction coefficient.

Micropipeline mechanical analysis is made as shown in Figure 4. According to the mechanical boundary conditions, equations can be obtained as follows:

$$
\left\{\begin{array}{l}
\tau=-\frac{\mathrm{d} N}{\mathrm{~d} x}, \\
\frac{\mathrm{d} Q}{\mathrm{~d} x}+q-P=0, \\
Q=\frac{\mathrm{d} M}{\mathrm{~d} x}-N \frac{\mathrm{d} \eta}{\mathrm{d} x},
\end{array}\right.
$$




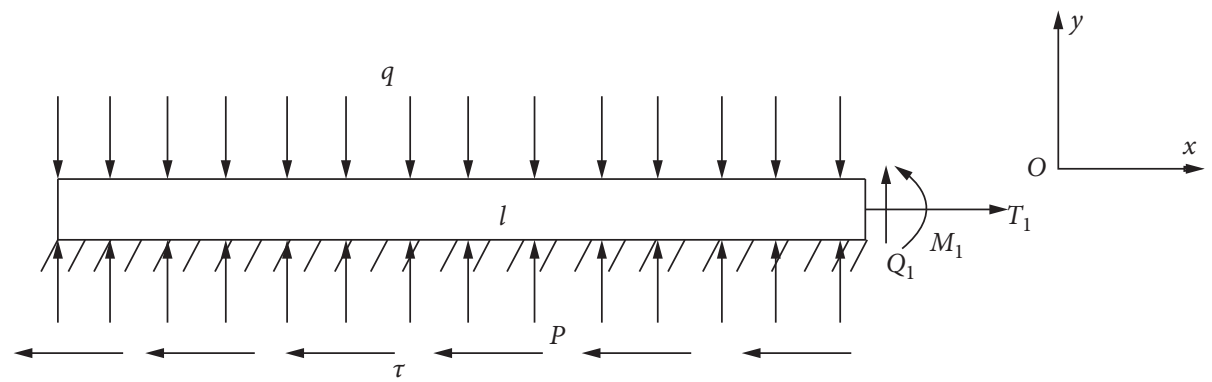

Figure 4: Pipeline mechanical model in sending ditch.

TABLE 1: Friction coefficient between various materials and soil [4].

\begin{tabular}{lc}
\hline Material and soil & $\mu$ \\
\hline Steel-sandy soil & $0.4 \sim 0.5$ \\
Steel-pebble & 0.25 \\
Concrete-sand & $0.35 \sim 0.55$ \\
Concrete-rubble & $0.6 \sim 0.65$ \\
\hline
\end{tabular}

where $Q$ is the shear force of the pipe section, $\mathrm{N} ; N$ is the support force of soil to the pipeline, $\mathrm{N}$; and $q$ is the gravity of the unit length of the pipeline, $\mathrm{N}$.

According to equation (5) and material mechanics formula $M=E I\left(\partial^{2} \eta / \partial x^{2}\right)$, the control differential equation of the pipeline in the sending ditch can be obtained:

$$
E I \frac{\partial^{4} \eta}{\partial x^{4}}-N \frac{\partial^{2} \eta}{\partial x^{2}}+\tau \frac{\partial \eta}{\partial x}+k_{0} D \eta+q=0
$$

Assuming that the deformation curve of pipeline in the sending ditch satisfies the Chebyshev polynomial,

$$
\eta=C_{1} x+C_{2}\left(2 x^{2}-1\right)+C_{3}\left(4 x^{3}-3 x\right)+C_{4}\left(8 x^{4}-8 x^{2}+1\right) .
$$

Combine with equations (6) and (7), the internal residual are obtained as follows:

$$
R_{1}=\sum_{i=1}^{5} A_{i} x^{i-1}
$$

where

$$
\begin{aligned}
A_{5}= & 8 C_{4} D k_{0}, \\
A_{4}= & 4 C_{3} D k_{0}+32 C_{4} \tau, \\
A_{3}= & 2 C_{2} D k_{0}-8 C_{4} D k_{0}+12 C_{3} \tau-96 C_{4} N, \\
A_{2}= & C_{1} D k_{0}-3 C_{3} D k_{0}+4 C_{2} \tau-24 C_{3} T-16 C_{4} \tau, \\
A_{1}= & C_{2} D k_{0}+C_{4} D k_{0}+192 C_{4} E I+C_{1} \tau-4 C_{2} T \\
& -3 C_{3} \tau+16 C_{4} T+q .
\end{aligned}
$$

According to pipeline boundary conditions, the constant coefficient of the deformation curve can be obtained as follows:

$$
\begin{aligned}
& C_{1}=\frac{8 L_{4}\left(4 L_{4}^{2}+3\right) d}{8 L_{4}^{4}+24 L_{4}^{2}+3}, \\
& C_{2}=\frac{4 d\left(6 L_{4}^{2}+1\right)}{8 L_{4}^{4}+24 L_{4}^{2}+3}, \\
& C_{3}=\frac{8 d L_{4}}{8 L_{4}^{4}+24 L_{4}^{2}+3}, \\
& C_{4}=\frac{d}{8 L_{4}^{4}+24 L_{4}^{2}+3} .
\end{aligned}
$$

2.2. Mechanical Model of Lifting Pipeline. During construction of the lifting pipeline, the gondola divides the pipeline into several segments, and the continuity conditions are satisfied between the adjacent spanning pipelines. Each spanning pipeline satisfies the second-order continual beam model, and the mechanical model is shown in Figure 5.

Each spanning pipeline is divided at the gondola, and each spanning pipeline is solved separately. The gondola can be equivalent to the movable hinge support in vertical direction, and the mechanics model of each spanning pipeline is shown in Figures 6-8.

Axial forces, bending moments, shear forces, and lateral uniform loads exist for each spanning pipeline, and the mechanical models have similarities. Take any micropipeline as the research object, and the mechanical model is shown in Figure 9.

The coordinate system of the whole mechanical model is oxy. According to the mechanical equilibrium conditions of micropipeline, the following equations can be obtained:

$$
\left.\begin{array}{l}
T+\Delta T-T=0 \\
Q+\Delta Q-Q-q \mathrm{~d} x=0 \\
M+\Delta M+\frac{1}{2} q(\mathrm{~d} x)^{2}+Q \mathrm{~d} x-M-T \mathrm{~d} y=0
\end{array}\right\} .
$$

By solving the model, the general solution of the pipeline deflection for each spanning pipeline can be obtained as follows: 
TABLE 2: Ground reaction coefficient $k_{0}\left(\mathrm{MN} / \mathrm{m}^{3}\right)[6]$.

I Loose soil (plastic clay, sand silt clay, and sandy and argillaceous sand, with organic sludge or peat soil II, III class by a thin layer of soil) $<30$

II $\quad$ Medium strength soil (clay, sand, and silt that approach plastic limit) 30 50

III Stiff soil (hard and sandy clay, gravel, and yellow soil) 50 100

IV Rock $>100$
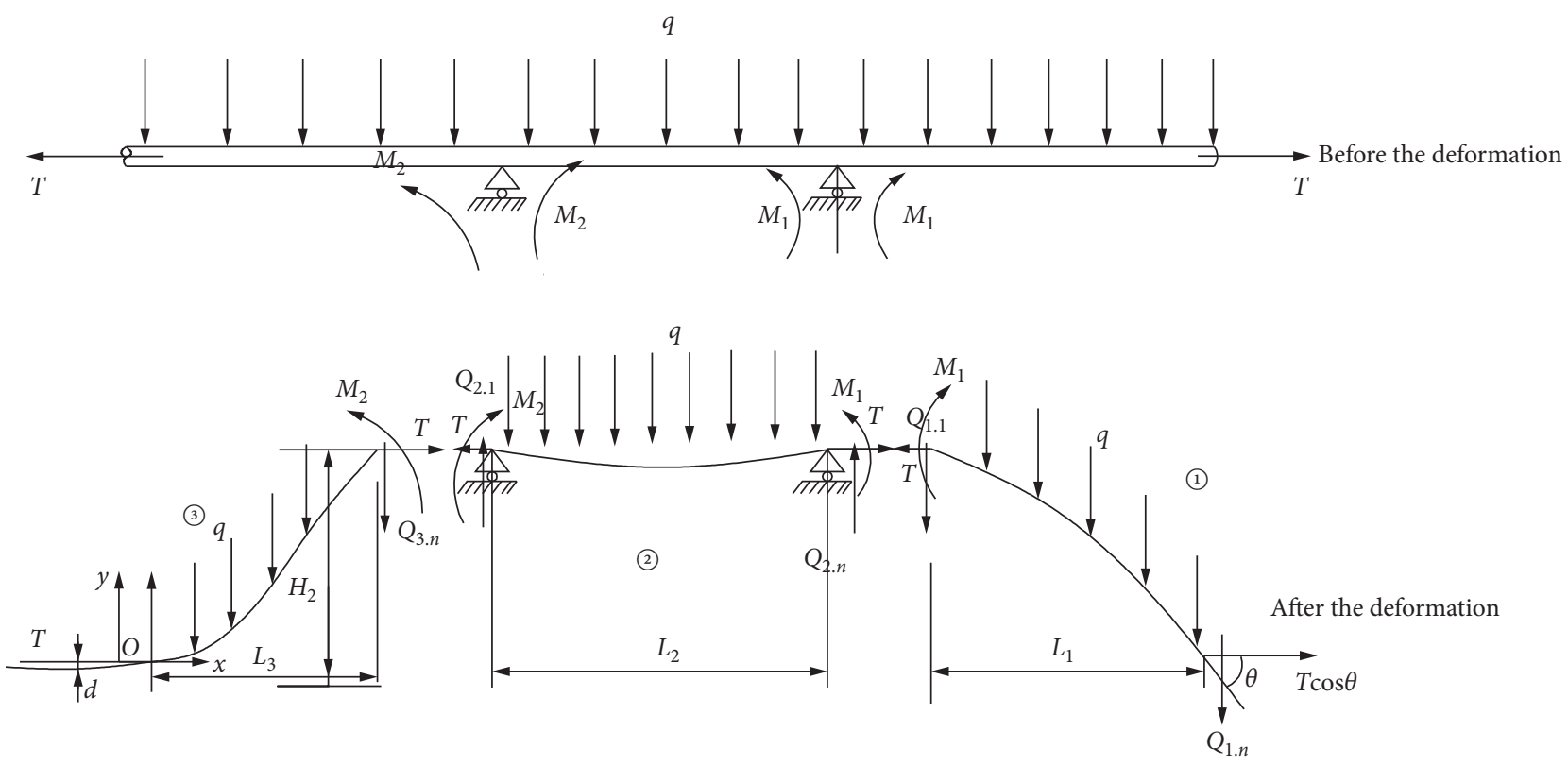

FIGURE 5: Mechanical model of lifting pipeline.

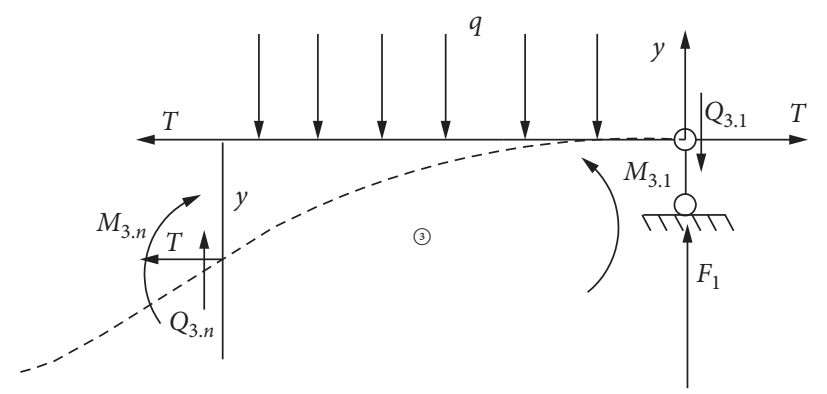

Figure 6: Pipeline mechanical model between the sending ditch and gondola 2.

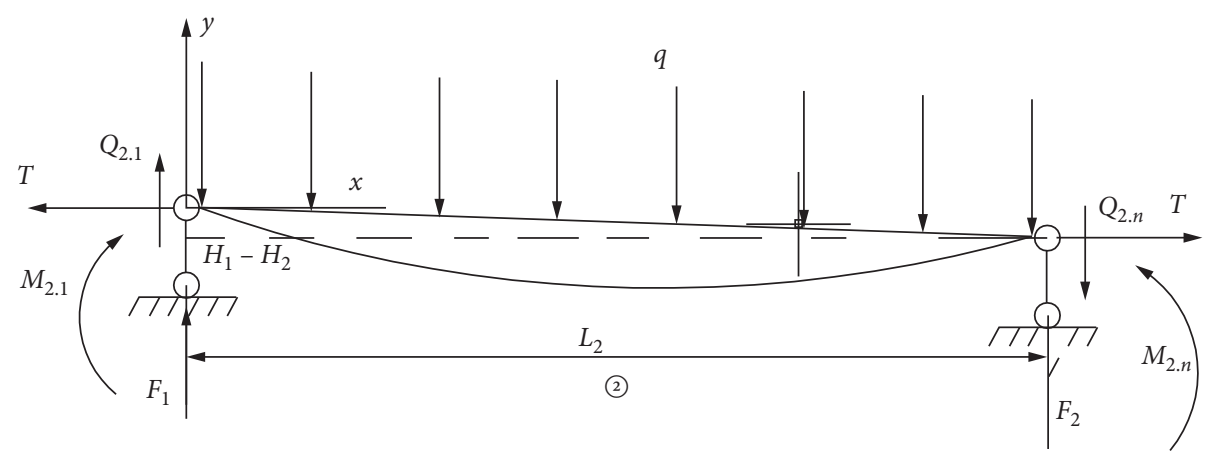

FIGURE 7: Spanning pipeline mechanical model between gondolas. 


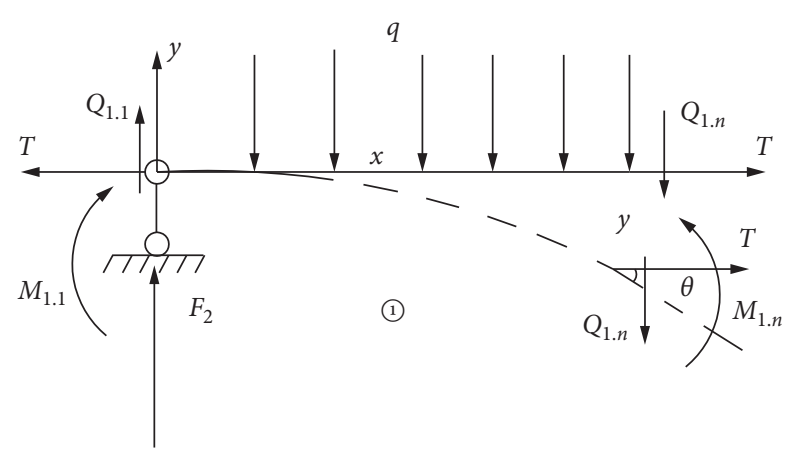

FIgURE 8: Pipeline mechanical model near the hole's inlet.

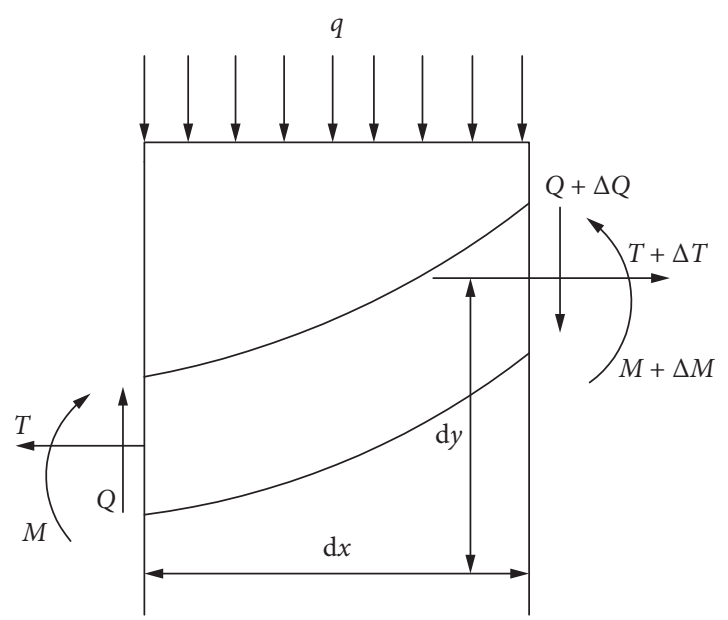

FIgURE 9: Micropipeline's mechanic model.

$$
\begin{aligned}
y_{i}= & C_{i 1} e^{(\sqrt{(T / E I)} x)}+C_{i 2} e^{(-\sqrt{(T / E I)} x)} \\
& +\frac{q}{2 T} x^{2}+C_{i 3} x+C_{i 4}, \quad i=1,2,3,
\end{aligned}
$$

where $C_{i 1}, C_{i 2}, C_{i 3}, C_{i 4}$ is an integral constant; $i$ represents the number of the spanning pipeline, 1 represents the spanning pipeline near the hole, 2 represents the spanning pipeline between the two gondolas, and 3 represents the spanning pipeline near the sending ditch; and $T$ is the pipeline axial force.

The pipeline between the sending ditch and gondola satisfies the continuity condition at the contact position, and the continuity equations are as follows:

$$
\left\{\begin{array}{l}
\left.y_{3}\right|_{x=0}=\left.\eta\right|_{x=0}, \\
\left.y_{3}^{\prime}\right|_{x=0}=\left.\eta^{\prime}\right|_{x=0}, \\
\left.y_{3}^{\prime \prime}\right|_{x=L_{3}}=\left.\eta^{\prime \prime}\right|_{x=0}, \\
\left.y_{3}^{\prime \prime \prime}\right|_{x=0}=\left.\eta^{\prime \prime \prime}\right|_{x=0} .
\end{array}\right.
$$

The frictional force between the pipeline and the gondolas is not considered, and the axial force of all pipelines is equal. According to the mechanical model shown in
Figure 7, the mechanical boundary conditions and continuity conditions at the gondola are as follows:

$$
\left\{\begin{array}{l}
\left.y_{3}\right|_{x=L_{3}}=\left.y_{2}\right|_{x=L_{3}}, \\
\left.y_{3}^{\prime}\right|_{x=L_{3}}=\left.y_{2}^{\prime}\right|_{x=L_{3},} \\
\left.y_{3}^{\prime \prime}\right|_{x=L_{3}}=\left.y_{2}^{\prime \prime}\right|_{x=L_{3}}, \\
\left.E I y_{3}^{\prime \prime \prime}\right|_{x=L_{3}}=\left.E I y_{2}^{\prime \prime \prime}\right|_{x=L_{3}}+F_{2},
\end{array}\right.
$$

where $F_{2}$ is the concentration force of gondola 2 on the pipeline.

It is necessary to ensure that the rotation angle of the pipeline is equal to the hole's entry angle to ensure that the pipeline smoothly enters into the hole. The rotation angle $\theta$ of the pipeline is related to the lifting height $H_{1}$ of the gondola 1 , the length of spanning pipeline $L_{1}$, and pipeline stiffness EI. However, there is a lack of practical theoretical support for the field construction, and most of the field construction adopts the experience method to adjust a variety of lifting parameters. The lifting parameters of the pipeline have different rotation angles, and the schematic diagram of parameters relationship is shown in Figure 10.

In the position of gondola 1 , the continuity condition of pipeline should be satisfied. The deflection of pipeline at the position of hole's inlet is 0 . Assume that the pipeline's rotation angle is $\theta$ (this angle is not equal to the entry angle). According to the mechanical model shown in Figure 8, the boundary and continuity conditions of spanning pipeline are as follows:

$$
\left\{\begin{array}{l}
\left.y_{2}\right|_{x=L_{2}+L_{3}}=\left.y_{1}\right|_{x=L_{2}+L_{3},}, \\
\left.y_{2}^{\prime}\right|_{x=L_{2}+L_{3}}=\left.y_{1}^{\prime}\right|_{x=L_{2}+L_{3},}, \\
\left.y_{2}^{\prime \prime}\right|_{x=L_{2}+L_{3}}=\left.y_{1}^{\prime \prime}\right|_{x=L_{2}+L_{3}}, \\
\left.E I y_{2}\right|_{x=L_{2}+L_{3}}=\left.E I y_{1}\right|_{x=L_{2}+L_{3}}+F_{1}, \\
\left.y_{1}\right|_{x=L_{1}+L_{2}+L_{3}}=0, \\
\left.y_{1}^{\prime}\right|_{x=L_{1}+L_{2}+L_{3}}=\theta,
\end{array}\right.
$$

where $F_{1}$ is the concentration force of the gondola 1 to the pipeline, $\mathrm{N}$ and $\theta$ is the pipeline's rotation angle, ${ }^{\circ}$.

\section{Equation Solving}

If the entry angle of the hole is $\theta_{0}$, then $\theta_{0}$ is a constant on the construction field. Only adjusting pipeline lifting parameters can smooth transition at the hole's inlet be satisfied as far as possible. The rotation angle of the pipe section is the first derivative of deflection. If the rotation angle $\theta$ and the entry angle $\theta_{0}$ are within a certain difference $\varepsilon$, the pipeline can smoothly enter into the hole, that is, it satisfies

$$
\left|y^{\prime}\right|_{x=L_{1}+L_{2}+L_{3}}-\theta_{0} \mid \leq \varepsilon
$$

where $\varepsilon$ is the allowable difference between the rotation angle of the pipeline and the entry angle of the hole, .

The concentrated force of the pipeline at the gondola, the relationship between the lifting height of pipeline, and the entry angle of the hole can be obtained by solving equations (1) (16). 


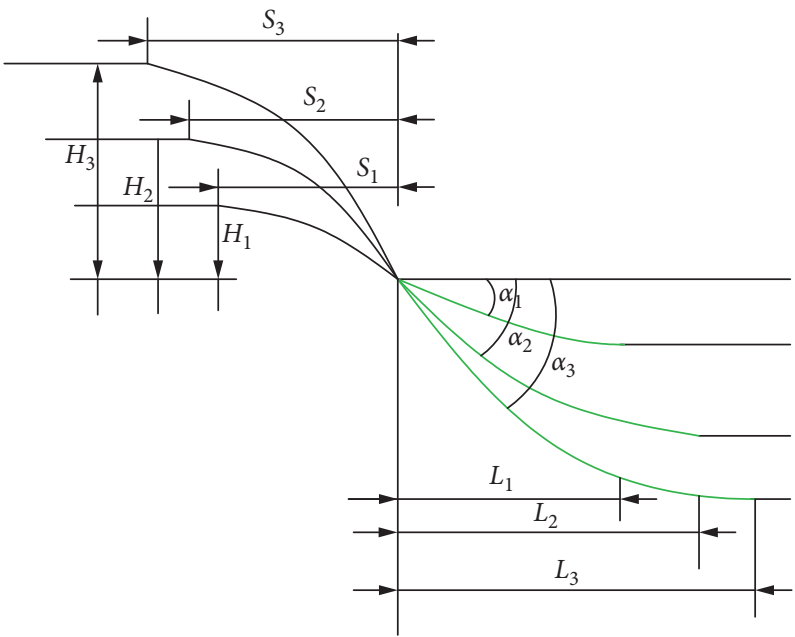

FIGURE 10: Schematic diagram of the relationship between the entry angle and lifting parameters.

The relationship between the lifting heights $H_{1}$ and $H_{2}$ of the gondolas and the lengths $L_{1}, L_{2}$, and $L_{3}$ of spanning pipeline are as follows:

$$
\left.\begin{array}{l}
H_{1}=C_{11} e^{\sqrt{(T / E I)} L_{1}}+C_{12} e^{-\sqrt{(T / E I)} L_{1}}+\frac{q L_{1}^{2}}{2 T}+C_{13} L_{1}+C_{14} \\
H_{2}=C_{31} e^{\sqrt{(T / E I)} L_{3}}+C_{32} e^{-\sqrt{(T / E I)} L_{3}}+\frac{q L_{3}^{2}}{2 T}+C_{33} L_{1}+C_{34}
\end{array}\right\} .
$$

Taking a field construction as an example, the pipe diameter is $813 \mathrm{~mm}$, the thickness of the pipe section is $8 \mathrm{~mm}$, and the stiffness of pipeline is $6.749 \times 10^{8}$. The friction coefficient between the pipe and soil is 0.45 . The unit length of pipeline's gravity is $q=634.96 \mathrm{~N} / \mathrm{m}$. By solving the abovementioned equation, the relationship between the lifting height and the length of pipeline and the axial force and the entry angle can be obtained as shown in Figures 11 and 12.

Through theoretical analysis, the relationship between the lifting height of gondola 2 and the length of the spanning pipeline can be obtained. When the length of spanning pipeline is small, the lifting height of gondola 2 is approximately 0 due to the pipeline stiffness. With increase in the length of pipeline, the lifting height of gondola 2 has a curve relationship with the spanning length $L_{3}$. The lifting height of gondola 2 varies linearly with unit length gravity $q$ and the axial force $T$. Under the same lateral load and axial force, the longer the spanning length is, the higher the lifting height of gondola 2 is. Under certain axial force, the longer the spanning length is, the higher the lifting height is. Under certain spanning length, the smaller the pipeline stiffness is, the higher the lifting height of gondola 2 is. The stiffness of oil and natural gas pipelines is between $3 \times 10^{8}$ and $7 \times 10^{8}$. Therefore, the lifting height of the gondola 2 is about $1 \sim 3.5 \mathrm{~m}$. Under the same pipe stiffness, the longer the suspension length is, the higher the lifting height of gondola 2 is.

The relationship between the lifting height of gondola 1 , spanning length $L_{1}$, pipeline rotation angle $\theta$, axial force $T$, and pipeline stiffness $E I$ is shown in Figures 13 and 14 .
The lifting height of the gondola 1 increases linearly with the entry angle of the hole. The larger the entry angle of the hole is, the higher the lifting height of the gondola is. The difference within a certain range between the rotation angle of pipeline and the entry angle of the hole can satisfy the pipeline smoothly entering into the hole. That is, under certain conditions of the entry angle, a certain lifting height range can satisfy the requirements of lifting construction. Under certain conditions of other parameters, the longer the pipe spanning length is, the higher the lifting height of the gondola is. With the same spanning length, the larger the entry angle is, the higher the lifting height is. The lifting height of the gondola has a curve relationship with the axial force. The larger the axial force is, the faster the lifting height changes with the axial force. Under the same axial force, the longer the suspension length is, the higher the lifting height of gondola 1 is. The lifting height of gondola 1 has a curve variation relation with the pipeline stiffness. The greater the stiffness is, the smaller the lifting height is. The pipeline stiffness varies from $5 \times 10^{8}$ to $7 \times 10^{8}$, and the lifting height of the gondola is about $3 \sim 4 \mathrm{~m}$. Under the same pipeline stiffness, the longer the spanning pipeline is, the higher the lifting height is.

According to the continuity beam theory, under a condition that the entry angle is known, the parameters such as the lifting height and the pipeline spanning length can be designed by theoretical calculation to satisfy the entry angle of the hole, so as to ensure that the pipeline smoothly enters into the hole.

\section{Strength Check}

In the lifting construction field of pipeline, in addition to ensuring that the pipeline smoothly enters the hole, to meet the pipe strength requirement is also important. It can be seen from Figure 5 that the stress on any pipe section is mainly the bending stress and axial tensile stress. The most dangerous position is the pipe section at the gondola, where the bending moment of the pipeline is the largest, so the 


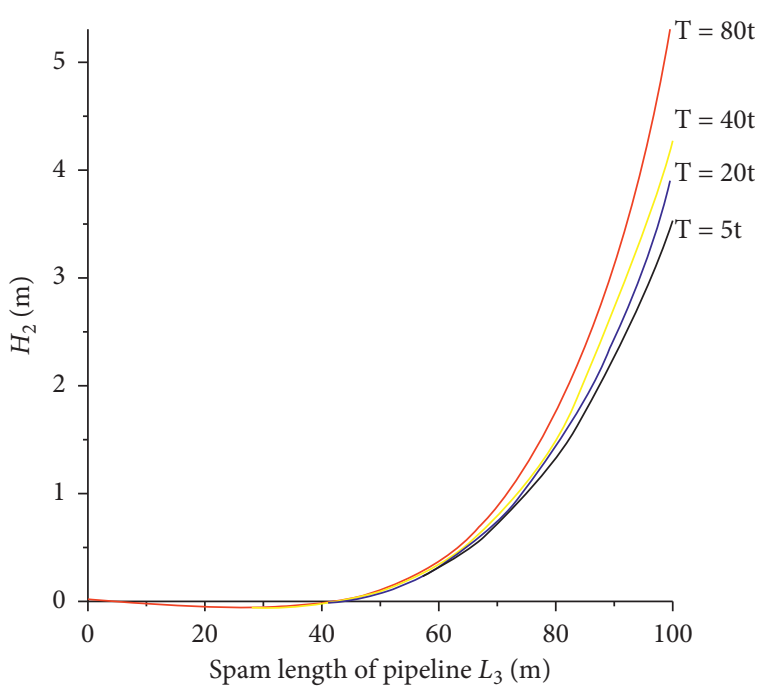

(a)

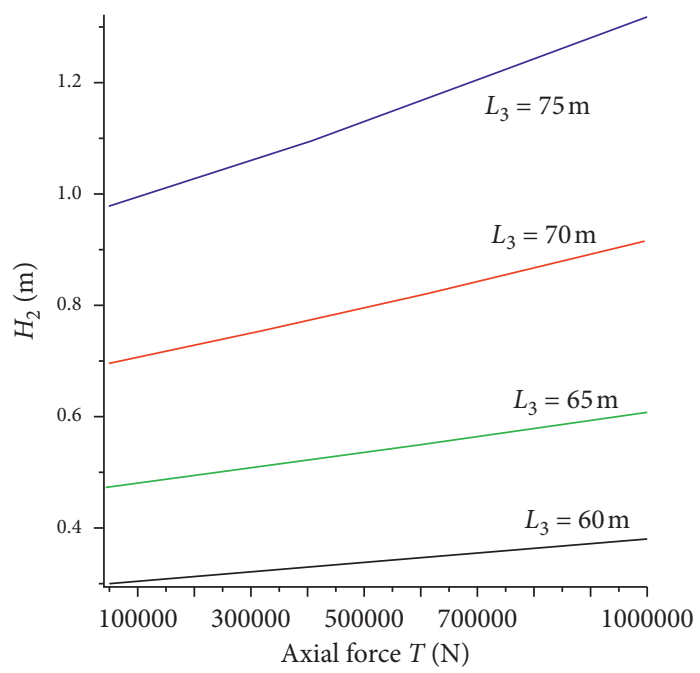

(b)

FIGURE 11: Lifting height of the gondola 2 varies with length of the pipeline and axial force. (a) Relationship between the lifting height and span length. (b) Relationship between the lifting height and axial force.

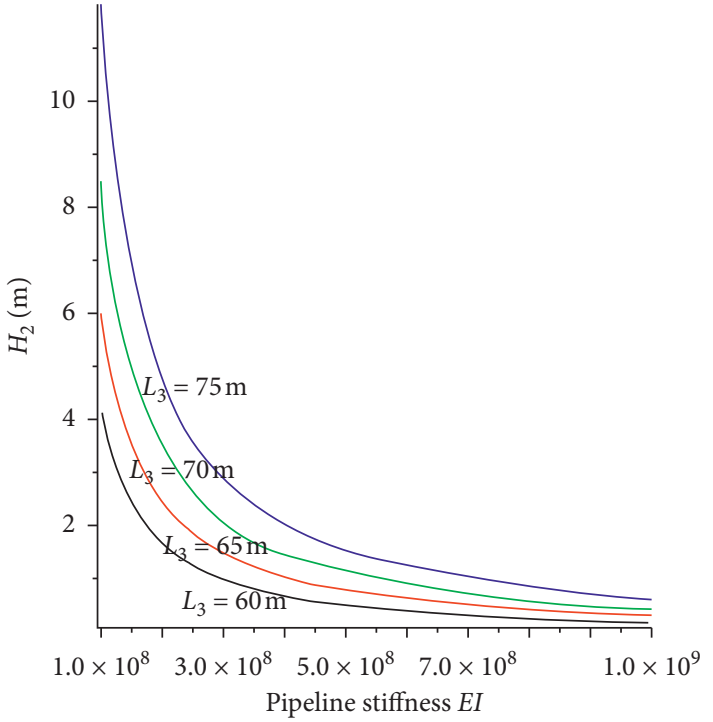

(a)

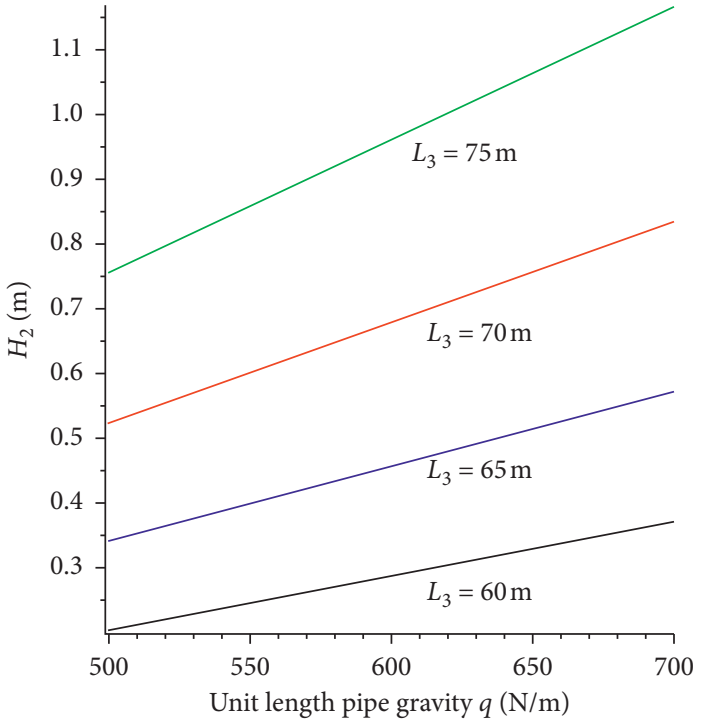

(b)

FIGURE 12: (a) Relationship between the lifting height of gondola 2 and stiffness. (b) Relationship between the lifting height of gondola 2and gravity.

stress is also the largest. Therefore, it is necessary to check the pipeline strength. The stress on the pipe section is mainly tensile stress and compressive stress. Therefore, only the maximum tensile stress or compressive stress of the pipeline should not exceed the tensile or compressive strength of the material. The lifting height of the pipeline cannot be lifted infinitely. If the lifting height of pipeline is too high, the bending tensile stress or compressive stress of pipeline may be too large and damage may occur.

It can be seen from Figure 5 that the maximum bending moment of the pipeline at the gondola is $M$. The pipe diameter is $D$, and the inertia moment of the pipeline is $I_{z}$. The maximum bending stress of pipeline at the gondola can be obtained as follows:

$$
\sigma=\frac{M}{I_{z}}
$$

The axial stress caused by the axial tensile force of the pipeline is as follows:

$$
\sigma_{T}=\frac{T}{A} .
$$

According to formulas (18) and (19), the maximum axial stress of the pipeline can be obtained: 


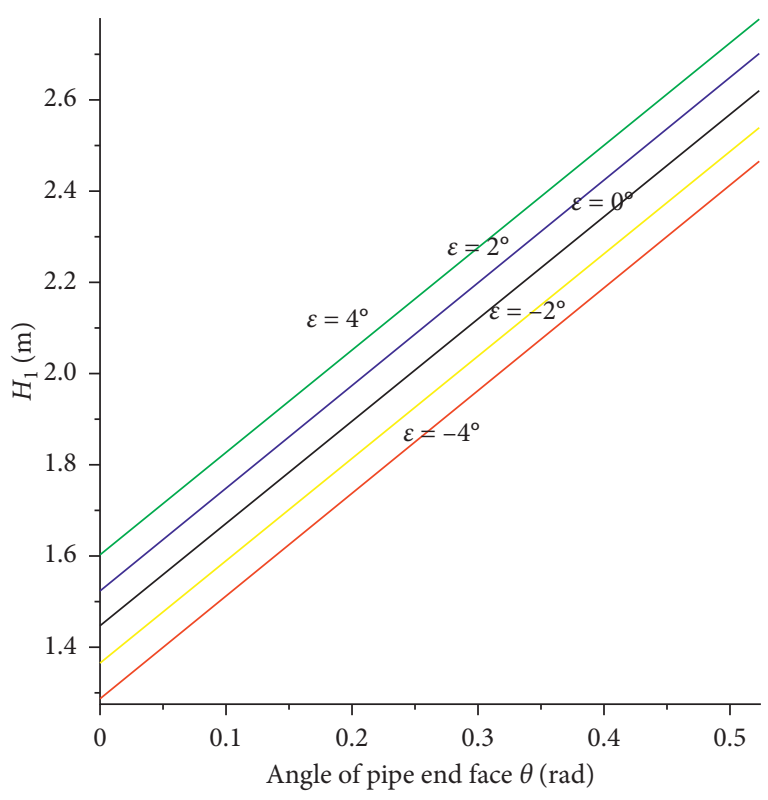

(a)

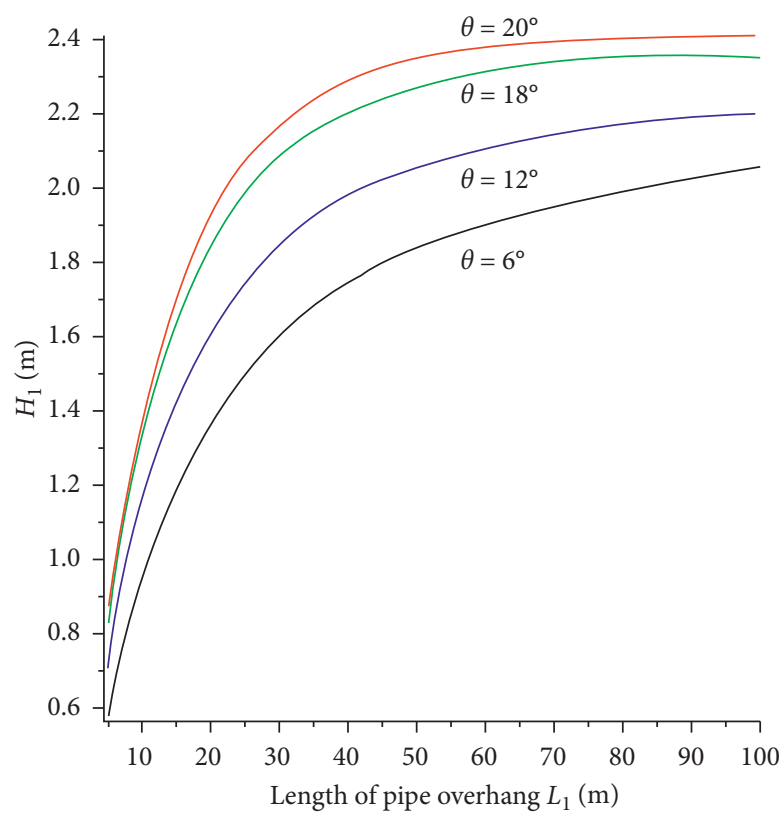

(b)

Figure 13: (a) Relationship between the lifting height of pipeline and the angle of end face. (b) Relationship between the lifting height of pipeline and the length of overhang.

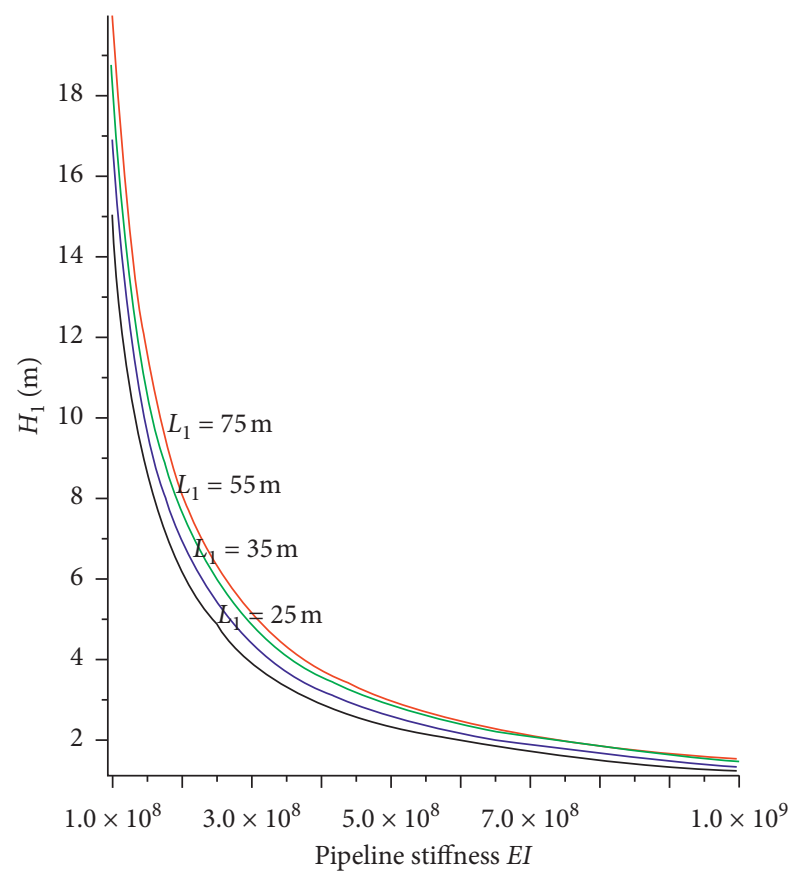

(a)

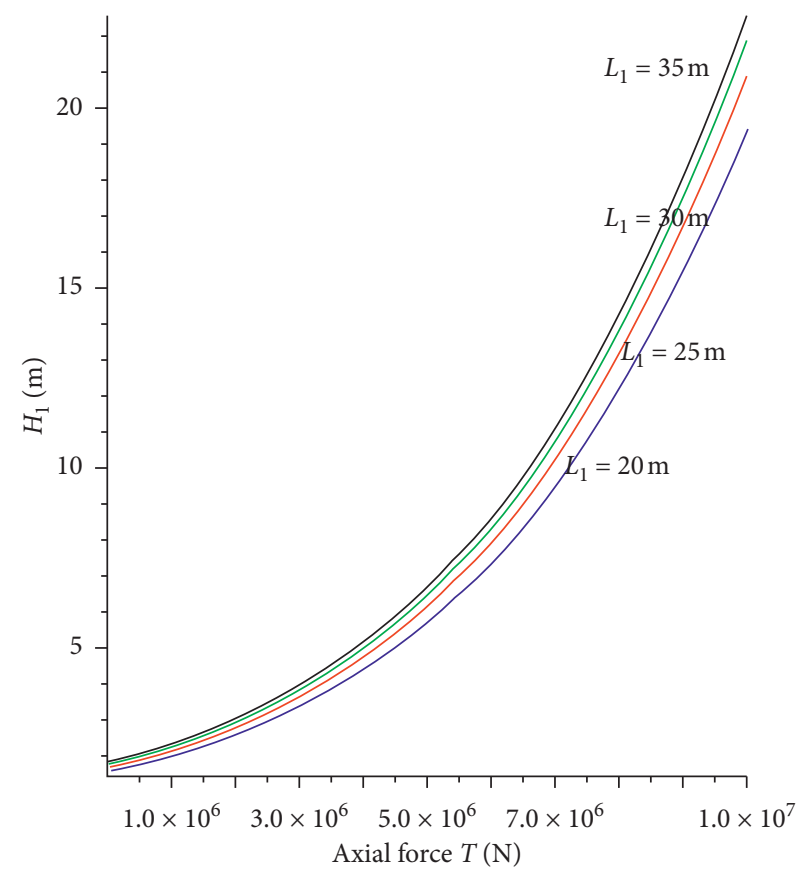

(b)

Figure 14: (a) Relationship between the lifting height of gondola 1and stiffness of pipeline. (b) Relationship between axial force and lifting height.

$$
\sigma_{\max }=\sigma+\sigma_{T}=\frac{\mu q L_{4}}{A}+\frac{M}{I_{z}},
$$

where $A$ is the pipe section area, $\mathrm{m}^{2}$, and $I_{z}$ is the pipe inertia moment, $\mathrm{m}^{4}$.

The fourth strength theory is used to check whether the strength of pipeline at the position of gondola is sufficient. 


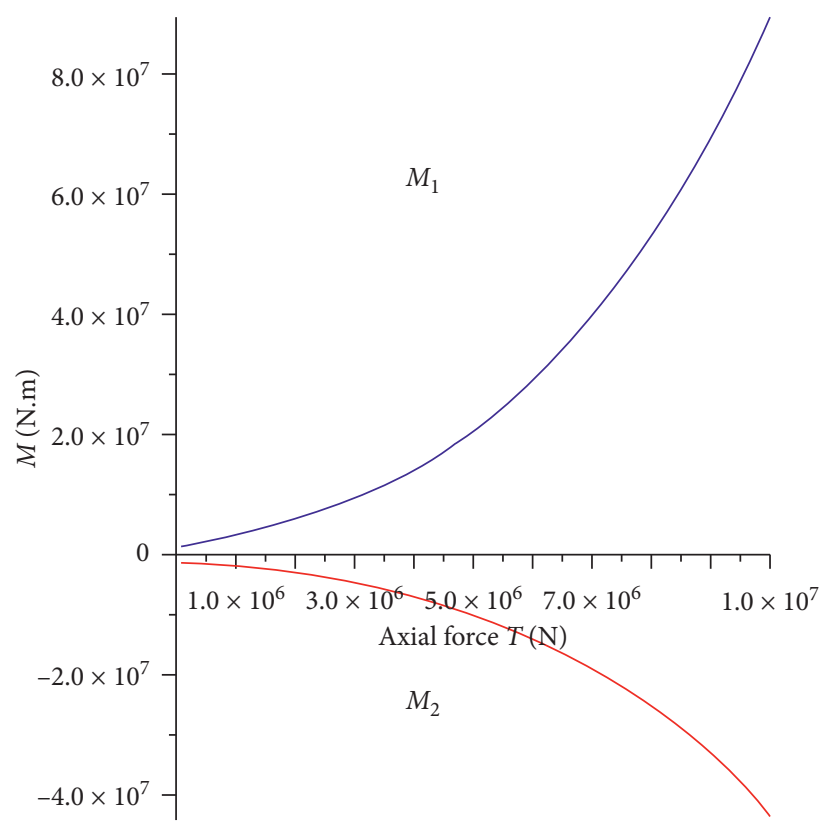

(a)

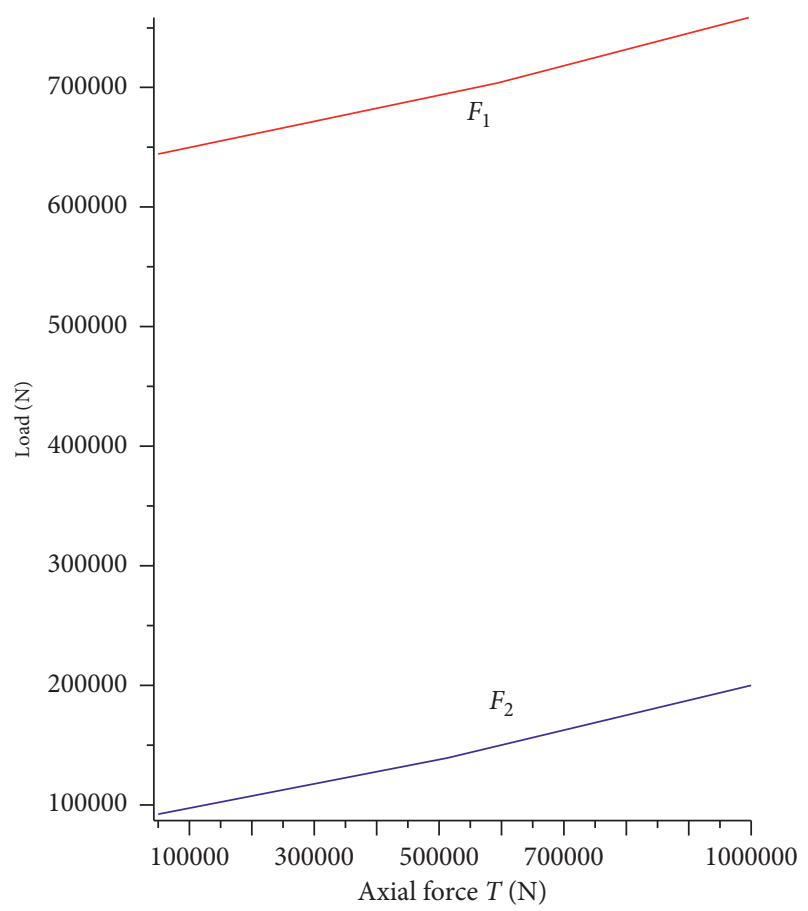

(b)

FIGURE 15: (a) Relationship between the bending moment and axial force. (b) Relationship between the lifting load and axial force.

When the axial stress and shear stress of the pipe section are known, it can be obtained:

$$
\left(\sigma_{r 4}\right)_{\max }=\left(\sqrt{\sigma_{\max }+3 \tau^{2}}\right)_{\max } \leq[\sigma]
$$

where $[\sigma]$ is the pipeline allowable stress, $\mathrm{Pa} ; \sigma_{\max }$ is the maximum axial stress, $\mathrm{Pa}$; and $\tau$ is the shear stress, $\mathrm{Pa}$.
Since the axial stress of the pipeline at the two gondolas is the same, the influence of the pipe stress is mainly the pipe bending stress. The bending moments of the pipeline at the position of gondolas 1 and 2 are as follows: follows:

$$
\left.\begin{array}{l}
M_{2}=\left.E I y_{3}^{\prime \prime}\right|_{x=L_{3}}=E I\left(c_{31} \frac{T}{E I} e^{\sqrt{(T / E I)} L_{3}}+c_{32} \frac{T}{E I} e^{-\sqrt{(T / E I)} L_{3}}+\frac{q}{T}\right) \\
M_{1}=\left.E I y_{1}^{\prime \prime}\right|_{x=L_{2}+L_{3}}=E I\left(c_{11} \frac{T}{E I} e^{\sqrt{(T / E I)}\left(L_{2}+L_{3}\right)}+c_{12} \frac{T}{E I} e^{-\sqrt{(T / E I)}\left(L_{2}+L_{3}\right)}+\frac{q}{T}\right)
\end{array}\right\} .
$$

The pipeline shear forces at the gondolas 1 and 2 are as

$$
\begin{aligned}
& Q_{2}=\left.E I y_{3}^{\prime \prime \prime}\right|_{x=L_{3}}=E I\left(c_{31} \frac{T}{E I} \sqrt{\frac{T}{E I}} e^{\sqrt{(T / E I)} L_{3}}-c_{32} \frac{T}{E I} \sqrt{\frac{T}{E I}} e^{-\sqrt{(T / E I)} L_{3}}\right) \\
& \left.Q_{1}=\left.E I y_{1}^{\prime \prime \prime}\right|_{x=L_{2}+L_{3}}=E I\left(c_{11} \frac{T}{E I} \sqrt{\frac{T}{E I}} e^{\sqrt{(T / E I)}\left(L_{2}+L_{3}\right)}-c_{12} \frac{T}{E I} \sqrt{\frac{T}{E I}} e^{-\sqrt{(T / E I)}\left(L_{2}+L_{3}\right)}\right)\right\} . \\
& \left.\begin{array}{l}
Q_{3 n}+F_{1}=Q_{21} \\
Q_{2 n}+F_{2}=Q_{11}
\end{array}\right\}
\end{aligned}
$$

The relationship between the shear forces at both ends of the gondola and the load of gondola are as follows: 


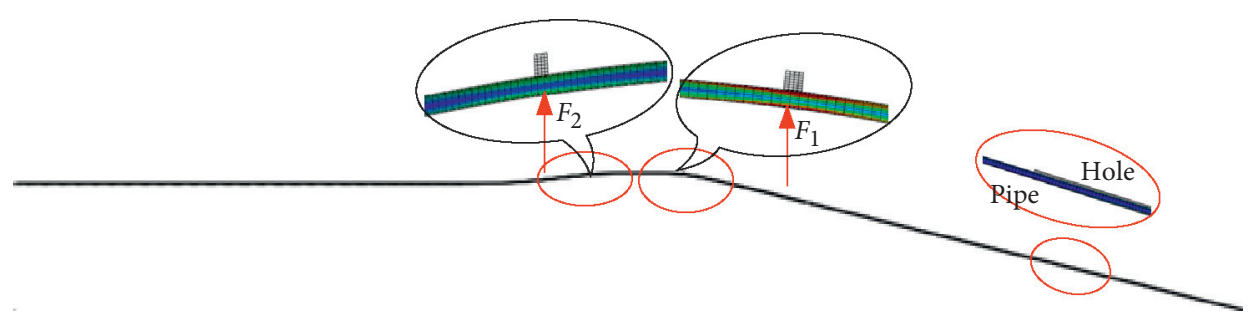

FIgURE 16: Numerical calculation model of pipeline hoisting.

TABle 3: Pipe material parameters.

\begin{tabular}{lcccc}
\hline Pipe material parameters & Density $\rho\left(\mathrm{kg} / \mathrm{m}^{3}\right)$ & Elasticity modulus $E\left(\times e^{11} \mathrm{~Pa}\right)$ & Poisson's ratio $(\mu)$ & Yield strength $\left(\delta_{0.05} \mathrm{MPa}\right)$ \\
\hline Parameters values & 7850 & 2.06 & 0.3 & 605
\end{tabular}

Through equations (22) and (24), the bending moment and the load variation curve of the pipeline at the hanging gondola can be obtained as shown in Figure 15.

According to formulas (21), (22), and (23), the maximum equivalent stress of the pipeline at the gondola can be obtained. Only when the maximum stress of the pipeline does not exceed, the allowable stress of the pipeline can satisfy the strength requirement.

It is found from Figure 15 that the bending moment and shear forces of the pipeline near the hole change fastest, and it is larger than the pipe bending moment near the sending ditch. The load of the gondola near the entry hole is larger than the load of the gondola near the sending ditch. Therefore, the pipe section near the hole at the gondola belongs to the most dangerous position, and strength check is mainly for the location of pipeline.

\section{Result Verification}

In order to verify the correctness of the theoretical model, a numerical calculation model is established for pipeline lifting, pipe wall thickness $\delta=8 \mathrm{~mm}$, length of pipeline $L=650 \mathrm{~m}$, and pipe diameter $D_{2}=813 \mathrm{~mm}$, and the calculation model is shown in Figure 16.

Pipe material parameters are shown in Table 3.

The relationship between the rotation angle and the length of pipeline can be obtained by numerical calculation as shown in Figure 17.

According to Figure 17, it is found that the maximum error between the numerical calculation result and the theoretical calculation result is $6.3 \%$, which satisfies the engineering error requirement $(20 \%)$. During the pipeline lifting construction, the lifting height $H_{1}$ of the gondola 1 near the hole has a great influence on the rotation angle of pipeline. Through fitting the numerical calculation results, it can be obtained that the relationship between the rotation angle of pipeline and the lifting height $H_{1}$ of gondola 1 is approximately linear, and the equation can be expressed as follows: $H_{1}=0.3436 \theta$.

\section{Conclusions}

The mechanical model of pipeline lifting construction is established by a theoretical method, and the relationship

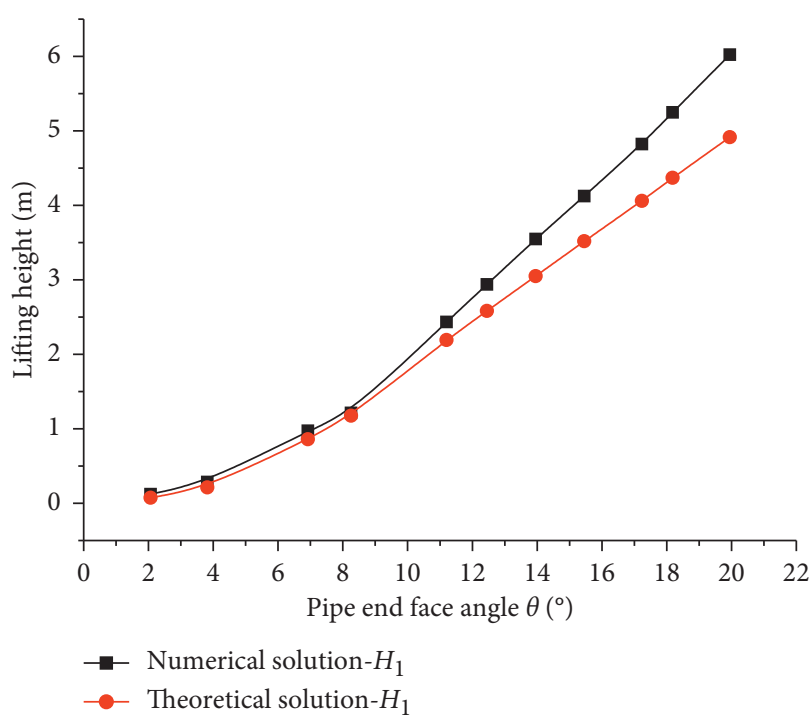

Figure 17: Comparison of theoretical and numerical solutions.

between parameters of lifting construction is obtained by the boundary conditions and continuity boundary conditions of each spanning pipeline. The following conclusions can be obtained:

(1) The lifting height of the gondola 1 varies linearly with the rotation angle of pipeline. The lifting height of gondola 1 varies linearly with the length of spanning pipeline. Under certain length of spanning pipeline, the larger the entry angle is, the higher the lifting height of the gondola 1 is.

(2) The lifting height of the gondola 1 has a curve variation with the stiffness and the axial force of pipeline. The larger the length of the spanning pipeline, the higher the lifting height of pipeline is required.

(3) The lifting height of the gondola 2 varies linearly with the gravity and axial force per unit length of pipeline. The lifting height of the gondola 2 varies with the axial force and the stiffness of pipeline in a curve. 
When the length of spanning pipeline is small, the lifting height of the gondola 2 is approximately 0 due to the pipeline stiffness.

The full-scale model of pipeline lifting construction is established by a numerical method, and the theoretical results are verified. The maximum error of the theoretical solution and the numerical solution is $6.3 \%$, which meets the requirements of engineering error in lifting pipeline and verifies the correctness of theoretical calculation results.

\section{Data Availability}

All data, models, and codes generated or used during the study are included within the article.

\section{Conflicts of Interest}

The authors declare that they have no conflicts of interest.

\section{Acknowledgments}

This work was supported by the Research Support Project of Chongqing University of Science and Technology (no. ckrc2019027).

\section{References}

[1] Z. Guo and Z. Liang, "Analysis of the pipeline pull-back load for horizontal directional drilling," KSCE Journal of Civil Engineering, vol. 22, no. 12, pp. 5133-5142, 2018.

[2] K. Yang, T. Ge, and X. Wang, "Attitude and position measurement of bit for underwater horizontal directional drilling system," Ocean Engineering, vol. 123, pp. 253-261, 2016.

[3] S. T. Ariaratnam, J. S. Lueke, and E. Anderson, "Reducing risks in unfavorable ground conditions during horizontal directional drilling," Practice Periodical on Structural Design \& Construction, vol. 9, no. 3, pp. 164-169, 2015.

[4] E. Cheng and M. A. Polak, "Theoretical model for calculating pulling loads for pipes in horizontal directional drilling," Tunnelling and Underground Space Technology, vol. 22, no. 56, pp. 633-643, 2007.

[5] J. S. Puckett, "Analysis of theoretical versus actual HDD pulling loads," in Proceedings of the New Pipeline Technologies, Security, and Safety, pp. 1346-1352, Baltimore, MD, USA, July 2003.

[6] C. Wu, G. Wen, X. Wu et al., "Calculation method of the pullback force for cable laying of the trenchless completed power pipeline," Journal of Coastal Research, vol. 73, pp. 681-686, 2015.

[7] L. Xu, A. Zhijiu, Q. Junchen, S. Wang, H. Qin, and H. Qian, "Mechanics analysis of pipe lifting in horizontal directional drilling," Journal of Natural Gas Science \& Engineering, vol. 31, pp. 272-282, 2016.

[8] Y. Ningping, Z. Jie, and H. Hanjing, "Status and development of directional drilling technology in coal mine," Procedia Engineering, vol. 73, pp. 289-298, 2014.

[9] H. Xia, H. Liu, R. Jiao, and K. Hu, "The decision support research of ultra-long distance pullback force computing model through the process of pipeline crossing pullback," Chaos, Solitons \& Fractals, vol. 89, pp. 527-532, 2016.

[10] F. Podbevsek, H. J. Brink, and J. Spiekhout, "Horizontal directional drilling: the influence of uplift and downlift during the pull-back operation," Journal of Pipeline Engineering, vol. 8, no. 4, 2009.

[11] S. Jeong, "Variability of subgrade reaction modulus on flexible mat foundation," Geomechanics and Engineering, vol. 13, no. 5, pp. 757-774, 2017.

[12] S. H. Lajevardi, D. Dias, and J. Racinais, "Analysis of soilwelded steel mesh reinforcement interface interaction by pullout tests," Geotextiles and Geomembranes, vol. 40, pp. 48-57, 2013.

[13] G. Xu, L. Cai, and Z. Wang, "Numerical simulation of pipesoil interaction during pulling back phase in horizontal directional drilling installations," Tunnelling and Underground Space Technology, vol. 76, pp. 194-201, 2018. 